Perturbations of the rectangular (equatorial) coordinates by Jupiter in units of the sixth decimal from 1854,0 to 1865.

\begin{tabular}{|c|c|c|c|}
\hline & $\xi$ & $\eta$ & $\zeta$ \\
\hline March 1,5 & -3977 & +3691 & +1992 \\
\hline 25,5 & -4454 & +3515 & +1935 \\
\hline April 18,5 & -4916 & +3322 & +1867 \\
\hline
\end{tabular}

MEAN ELEMENTS.

1854,0 Washington $M$. T.

$M=80^{\circ} 8^{\prime} 53^{\prime \prime} 3$

$\pi=15531,0\}$ M. Eq. Ep.

$\left.\Omega=\begin{array}{lll}150 & 3 & 49,7\end{array}\right\}$

$i=10916,9$

$\varphi=123420,2$

$\mu=1020^{\prime \prime} 1198^{\circ}$

$\log a=0,360993$.

\title{
Aus einem Schreiben des Herrn Oppolzer an den Herausgeber.
}

Der Comet I. 1862 hatte eine so rasche Bewegung, dass die bekannten Näherungsausdrücke für Berücksichtigung der Eigenbewegung bei Kreismikrometern nicht mehr völlig ausreichen. Folgende Formeln jedoch sind selbst bei den bisher gekannten stärksten Eigenbewegungen völlig ausreichend und sind nahezu streng. Ist $d \alpha$ und $d \delta$ die Eigenbewegung des Cometen in 1 Zeitsecunde, so bestimmt man den Hülfswinkel $i$ aus :

$$
\operatorname{tg} i=\frac{d \delta}{(15-\delta \alpha) \cos \delta}=\frac{d \delta}{N}
$$

Die Länge der Sehne, welche der Comet im Mikrometer beschreibt, ist alsdann :

$$
s=t(15-\delta \alpha) \frac{\cos \delta}{\cos i}=\frac{t N}{\cos i} .
$$

Nennt man den Abstand der Sebne rom Mittelpunkt $x$, so wird :

Wien, 1864 Mai 3.

$$
\begin{aligned}
\delta-\Delta & =x \cos i \\
\Delta x & =\frac{x \sin i}{\cos \delta} .
\end{aligned}
$$

Der Winkel $i$ ist, so lange $d \alpha<15$, was wohl hier nur in Betracht kommt, zwischen $+90^{\circ}$ und $-90^{\circ}$ zu zählen. Es ist also bei der Berechnung des Perpendikels und der Sehne nur darauf zu achten, ob der Comet, beziehungsweise Planet, nördlich oder südlich vom Kreismittelpunkte ging, da dem entsprechend $x=$ Perpendikel + und - zu nehmen ist; dies ist wegen der Correction in AR wichtig, da $\Delta \alpha=\frac{x \sin i}{\cos \delta}$ sonst leicht das unrichtige Zeichen erhalten würde ; bei

$$
(\delta-\Delta)=x \cos i
$$

ist die Berïcksichtigung des Zeichens von $x$ selbstverständlich.

\section{Theodor Oppolzer.}

\section{Aus einem Schreiben des Hern Ministerialraths von Steinheil an den Herausgeber.}

Als ich in 21472 der Astr. Nachr. das Schreiben des Herrn T'empel gelesen hatte, drängte sich mir die Vermuthung auf, dass die sogenannten falschen Sternchen, welche Herr Tempel an Sirius und mehreren anderen Sternen erster Grösse bemerkt hatle, in Auge des Beobachters ihren Ursprung haben müssen, da Reflexbilder im Oculare weder bei allen Lagen im Gesichtsfelde, noch bei verschiedenen Vergrösserungen ungeänderte Abstände vom Hauptstern gehen können. Ich liess daher Hern Tempel auffordern, darüher durch Beobachtungen zu entscbeiden, indern er beim Hineinsehen in das Fernrohr den Kopf etwas um die Absehensliuie drehen möge. Drehen sich dabei die falschen Sterne mit dent Auge, so haben sie auch im Auge ihren Ursprung. Nun antwortet beute Herr Tenipel unterm 18. Mai und bestätigt durch Beobachtung an Capella und Vega meine Vermuthung. Ich erfülle seinen Wunsch, indem ich Ihnen dies sogleich mitheile.

Prof. Dr. Donders in Utrecht theilte meinem Sohne Dr. Adolph Steinheil vor einiger Zeit mit, dass er an verschiedenen Augen schon Nebenbilder (solche, die nicht mit dem Hauptbilde zusammenfallen) beobachtet habe, und dass die Erscheinung ihren Grund in der Bildung der Krystallinse haben könne, weiche fächerförmige Abtheilungen (pomeranzenähnlich) nachweise.

Mü ach e a, 1864 Mai 21. 